\title{
Usinabilité à grande vitesse et à sec des couches du rechargement base-nickel par soudage d'outillage à chaud
}

\author{
Mohamed El Mansoria ${ }^{\mathrm{a}}$, Laurence Fouilland-Paillé et Fabrice Pierron \\ Laboratoire de Mécanique et Procédés de Fabrication (JE2381), École Nationale Supérieure d'Arts et Métiers - CER - Châlons, \\ Rue Saint Dominique, BP 508, 51006 Châlons en Champagne, France
}

Reçu le 15 janvier 2004, accepté le 5 mars 2004

\begin{abstract}
Résumé - Ce papier relate certains faits expérimentaux relatifs à l'usinabilité à grande vitesse des couches de rechargement base-nickel pour la fabrication d'outillages de travail à chaud. L'étude part des examens métallurgiques des couches rechargées et s'étend aux facteurs limitatifs de la coupe observés lors des essais de rainurage instrumentés. La limitation principale de ces rechargements à la coupe est liée à leur structure métallurgique fortement hétérogène. Cette hétérogénéité consiste en un réseau dendritique (phasenickel ductile) séparé par une dispersion interdendritique fragile (phase-carbures et phases finement ou grossièrement précipitées). Ceci se traduit en usinage par un écrouissage superficiel élevé à l'origine ensuite d'une usure prématurée des outils de coupe. Cette dégradation est dominée par une adhésion entretenue du copeau (frottement sec collant) conduisant à des entailles ainsi qu'un écaillage très sévères de l'outil de coupe consécutif à un effritement du matériau de coupe.
\end{abstract}

Mots clés : Usinabilité / usure / rechargement base-nickel

Abstract - Machinability at high speed and dry condition of nickel-base weld-hardfacing deposit for hot tooling. This paper reports some experimental findings concerning the machinability at high cutting speed of nickel-base weld-deposited hardfacings for the manufacture of hot tooling. The study begins with the metallurgical observation of the welded layers and extends to the factors impairing their machinability as observed from instrumented slotting tests. The primary limitation when machining these weld-hardfacing layers is related to their heterogeneous microstructure. This heterogeneity consists of a dendritic network (ductile nickel-phases) separated by a fragile interdendritic dispersion (carbides-phases and fine or coarse precipitates). This is expressed during machining by a high surface work-hardening involving a premature wear of the cutting tools. This degradation is dominated by an adhered chip (dry sticking friction) leading to notches as well as a very severe chipping of the cutting tool following crumbling of tool material.

Key words: Machinability / wear / nickel-base hardfacing

\section{Introduction}

Dans l'industrie des moules et matrices, une part importante des coûts de production est réservée au processus d'usinage car, généralement, d'importants volumes de matière sont enlevés. L'outillage fini est lui aussi soumis à des tolérances géométriques et d'état de surface très strictes. C'est la raison pour laquelle l'usinabilité du matériau d'outillage, son aptitude au polissage et ses propriétés thermomécaniques doivent être

a Auteur correspondant :

mohamed.elmansori@chalons.ensam.fr considérées avec attention afin de réaliser des économies de production maximales. Cependant, compte tenu du rôle prépondérant des opérations d'usinage, il est important de pouvoir bénéficier d'une bonne usinabilité d'ensemble. Les développements exposés dans ce travail font le point sur l'usinabilité des rechargements par soudage d'outillage pour la fabrication de matrices de forgeage à chaud et de moules de coulées sous pression. En effet, le rechargement est souvent pratiqué à titre préventif ou curatif et ce afin de prolonger la durée de vie d'outillages en service. Sa validation technico-économique est conditionnée par l'usinabilité ainsi que par la fiabilité en service du rechargement. Dans le cas des aciers d'outillage pour 


\section{Nomenclature}

\begin{tabular}{|c|c|c|}
\hline$a_{\mathrm{e}}$ & Engagement radial & $(\mathrm{mm})$ \\
\hline$a_{\mathrm{p}}$ & Engagement axial & $(\mathrm{mm})$ \\
\hline$a_{\mathrm{o}}$ & Rayon de la phase ductile & $(\mathrm{mm})$ \\
\hline$B$ & Épaisseur de l'éprouvette CT & $(\mathrm{mm})$ \\
\hline$C$ & Capacité thermique massique & $\left(\mathrm{J}^{\mathrm{kg}}{ }^{-1} \cdot{ }^{\circ} \mathrm{C}^{-1}\right)$ \\
\hline$D$ & Longueur du défaut & $(\mathrm{mm})$ \\
\hline$d$ & distance du substrat & $(\mathrm{mm})$ \\
\hline$E_{\mathrm{d}}$ & Module élastique de la phase ductile (phase nickel dendritique) & $(\mathrm{GPa})$ \\
\hline$e_{\mathrm{d}}$ & Épaisseur de rechargement usinée & $(\mathrm{mm})$ \\
\hline$f_{\mathrm{z}}$ & Épaisseur de copeau non déformée ou avance & $(\mathrm{mm})$ \\
\hline$h_{\mathrm{c}}$ & Épaisseur moyenne du copeau & $(\mathrm{mm})$ \\
\hline$K$ & Conductivité thermique & $\left(\mathrm{W} \cdot \mathrm{m}^{-1} \cdot{ }^{\circ} \mathrm{C}^{-1}\right)$ \\
\hline$K_{\mathrm{m}}$ & Ténacité de la matrice fragile interdendritique & $\left(\mathrm{MPa} \cdot m^{1 / 2}\right)$ \\
\hline$K_{\max }$ & Facteur d'intensité maximale de contrainte & $\left(\mathrm{MPa} \cdot m^{1 / 2}\right)$ \\
\hline$N$ & Fréquence de rotation de la broche & $\left(\operatorname{tr} . \min ^{-1}\right)$ \\
\hline$P$ & Charge compressive du contact pion-disque & $(\mathrm{N})$ \\
\hline$P_{\mathrm{c}}$ & Puissance en charge & $(\mathrm{W})$ \\
\hline$P_{\max }$ & Charge maximale appliquée & $(\mathrm{N})$ \\
\hline$P_{\mathrm{o}}$ & Puissance à vide & $(\mathrm{W})$ \\
\hline$r$ & Rapport de compression & \\
\hline$S$ & Distance séparant le défaut de la fissure & $(\mathrm{mm})$ \\
\hline$T_{i}$ & Durée de vie de l'outil n ${ }^{\circ} i$ & $(\min )$ \\
\hline$u$ & Énergie spécifique de coupe & $\left(\mathrm{J} . \mathrm{m}^{-3}\right)$ \\
\hline$V_{\mathrm{c}}$ & Vitesse de coupe & $\left(\mathrm{m} \cdot \min ^{-1}\right)$ \\
\hline$V_{\mathrm{f}}$ & Fraction volumique de la phase ductile & \\
\hline $\bar{V}_{\mathrm{g}}$ & Vitesse moyenne de glissement du copeau sur la face de coupe & $\left(\mathrm{m} \cdot \min ^{-1}\right)$ \\
\hline$W$ & Fréquence de rotation du disque & $\left(\right.$ tr.min $\left.{ }^{-1}\right)$ \\
\hline$z$ & Nombre de dents d'outil coupant & \\
\hline$\phi$ & Diamètre d'outil coupant & $(\mathrm{mm})$ \\
\hline $\bar{\theta}_{\mathrm{W}}$ & Température moyenne de la surface usinée à la sortie immédiate & \\
\hline & d'outil coupant & $\left({ }^{\circ} \mathrm{C}\right)$ \\
\hline$\rho$ & Masse volumique & $\left(\mathrm{kg} \cdot \mathrm{m}^{-3}\right)$ \\
\hline$\sigma_{y}$ & Limite élastique du rechargement & $\left(\mathrm{N} \cdot \mathrm{m}^{-2}\right)$ \\
\hline$\Delta K_{\mathrm{PF}}$ & Ténacité due au renforcement par pontage de fissure & $\left(\mathrm{MPa} \cdot \mathrm{m}^{1 / 2}\right)$ \\
\hline$\Delta K_{\mathrm{DF}}$ & Ténacité due au renforcement par courbure et déviation de fissure & $\left(\mathrm{MPa} . \mathrm{m}^{1 / 2}\right)$ \\
\hline$\chi$ & Travail à rupture de la phase ductile & $(\mathrm{N} . \mathrm{m})$ \\
\hline$\sigma_{\mathrm{o}}$ & Limite élastique de la phase ductile & $\left(\mathrm{N} \cdot \mathrm{m}^{-2}\right)$ \\
\hline$\theta$ & Angle de déviation & \\
\hline \multicolumn{3}{|c|}{ Abréviations : } \\
\hline $\mathrm{PCBN}$ & Nitrure de bore cubique polycristallin & \\
\hline PCD & Diamant polycristallin & \\
\hline PVD & Dépôt physique en phase vapeur & \\
\hline TAU & Tests accélérés d'usinabilité & \\
\hline TiAlN & Nitrure aluminium de titane & \\
\hline $\mathrm{MoS}_{2}$ & Bisulfure de molybdène & \\
\hline
\end{tabular}


travail à chaud, trois nuances de rechargement sous forme de superalliages à base de fer, de nickel ou de cobalt sont utilisées. La limitation essentielle des alliages à base de fer réside dans la température maximale autorisée en service (550 ${ }^{\circ} \mathrm{C}$ environ). Ainsi lorsqu'il y a élévation conjointe de température $\left(>550{ }^{\circ} \mathrm{C}\right)$ et de pénétration thermique (>1 mm), le rechargement à base-nickel ou à base-cobalt devient incontournable [1]. Néanmoins, leur usinabilité difficile représente un obstacle majeur à leur plus large diffusion. Partant de ce constat, l'étude que nous exposons ici a pour objectif de faire apparaître les mécanismes qui régissent le comportement à la coupe grande vitesse et à sec du rechargement à base-nickel. Des essais de coupe instrumentés en puissance, en effort et en température ont été effectués sur des blocs rechargés en superalliage basenickel. La présentation se décline ainsi en trois parties :

1. la première partie introduit quelques repères bibliographiques axés sur les facteurs limitatifs de la coupe des matériaux réfractaires;

2. la description des couches de rechargement étudiées, des techniques expérimentales et des moyens de mesure utilisés fait l'objet de la seconde partie ;

3. la troisième partie expose les résultats obtenus et précise quelques mécanismes utiles à la compréhension du comportement à la coupe des rechargements basenickel.

\section{2 Éléments bibliographiques}

L'ensemble des auteurs traitant de la coupe des superalliages base-nickel à dominante réfractaire s'accorde à considérer que l'usure prématurée de l'outil coupant constitue le facteur limitatif majeur de leur usinabilité. Celle-ci résulte de l'activation simultanée de certains phénomènes mécaniques et physico-chimiques lors de la coupe, formulés comme suit par Ezugwu et al. [2] dans un article de synthèse :

- la majeure partie de leur résistance mécanique est conservée durant la coupe en raison de leurs performances élevées à hautes températures ;

- la présence d'un fort durcissement par déformation (écrouissage) contribue largement à l'usure en entaille au bec d'outil et/ou sur l'arête tranchante;

- les arêtes de coupe sont soumises à une usure abrasive sévère due à la présence d'une forte dispersion de carbures ;

- la propension du matériau de coupe à se combiner chimiquement avec la matière usinée à des températures de coupe élevées conduit à une usure d'outil par diffusion;

- la soudure ou l'adhésion fréquente de ces matériaux aux outils de coupe conduisent à des entailles ainsi qu'à un écaillage très sévère de la face de coupe suite à un effritement du matériau de coupe;

- la formation de copeaux trempés et difficiles à contrôler lors de la coupe, contribue à une usure par cratérisation et par collage;
- la faible diffusivité thermique de ces matériaux génère une température élevée à la pointe d'outil ainsi que de forts gradients thermiques au sein du matériau de coupe.

On constate que, dans cette formulation, la durée de vie d'un outil coupant, pour un niveau de sollicitation donné, sera d'autant plus grande que le matériau de coupe combine [3] :

- une dureté à chaud élevée;

- une très bonne résistance à l'usure;

- une haute ténacité;

- une bonne endurance aux chocs;

- une stabilité chimique adéquate aux hautes températures.

Certaines de ces exigences sont difficilement conciliables, pour un même matériau de coupe, d'autant plus qu'elles doivent être conservées durant la coupe. De la comparaison des performances des outils coupants testés sur certains matériaux à caractéristiques réfractaires, il ressort que $[2,4]$ :

- les aciers rapides sont inadaptés pour la coupe de ces matériaux en raison de leurs faibles dureté et rigidité. Leur module d'Young relativement faible favorise les flexions et les vibrations en particulier pour les petits diamètres ;

- le cermet, lui, supporte très mal les chocs, les vibrations et la coupe en opposition. De plus, les outils de fraisage en cermet sont très coûteux;

- le nitrure de bore cubique polycristallin (PCBN) sous la forme de plaquettes brasées ou fixées mécaniquement est fragile, bien qu'il permette une augmentation importante de la vitesse de coupe et des durées de vie;

- les outils en diamant polycristallin (PCD) sont inappropriés à cause de la forte réactivité du diamant et de sa transformation en graphite pour des températures d'environ $750{ }^{\circ} \mathrm{C}$;

- les carbures de type P et K monoblocs avec un module d'élasticité élevé réduisent les vibrations de flexions. Les plaquettes amovibles conviennent mieux que les outils monoblocs ;

- les revêtements mono ou multicouches tels que TiN, TiCN, TiAlN, etc., sur des outils en carbure micrograin, obtenus par dépôt physique en phase vapeur (PVD) présentent des performances intéressantes et conduisent parfois à des résultats prometteurs.

Il est donc primordial d'avoir une idée aussi précise que possible sur les mécanismes d'enlèvement de matière du rechargement d'outillage base-nickel à propriétés réfractaires afin de mieux optimiser les opérations d'usinage de l'outillage fini. Néanmoins, si l'usinabilité des superalliages à base-nickel en matériaux massifs forgés ou coulés a suscité de nombreux travaux $[2,3,5]$, il n'a pas été trouvé dans la littérature d'études traitant directement la coupe de ces matériaux sous forme de couches rechargées par soudage. Il existe sûrement quelques données 
et savoir-faires non publiés et propres à certaines entreprises de forge. Ainsi, les éléments de bibliographie discutés ci-dessous serviront seulement comme indicateurs utiles à la compréhension de l'usinabilité des couches de rechargement base-nickel.

Cockerman [6] fut parmi les premiers à étudier l'influence des facteurs métallurgiques sur la résistance à la propagation des fissures dans différents rechargements réfractaires base-nickel obtenus par soudage. La ténacité du rechargement, c'est-à-dire le facteur critique d'intensité des contraintes amenant la décohésion, et la vitesse de propagation macroscopique ont été évalués par des essais de fissuration par fatigue sur des éprouvettes CT selon les recommandations préconisées par la norme ASTM E399 [7]. Les éprouvettes CT ont été méthodiquement taillées dans les trois couches supérieures du rechargement sur des échantillons présentant initialement cinq couches rechargées. L'auteur souligne que cette préparation d'éprouvette s'imposait afin d'éviter le phénomène de dilution auquel sont fortement assujetties les deux premières couches attenantes au substrat métallique. Les résultats de rupture ont montré que :

- d'une manière générale, la microstructure des rechargements base-nickel est fortement hétérogène et consiste en une matrice fragile interdendritique continue composée de phases carbures, borures, siliciures ainsi que des phases intermétalliques qui séparent le réseau dendritique composé essentiellement en phasenickel ductile;

- la possibilité de trouver des valeurs de ténacité valides est conditionnée par la relaxation de trois critères sur les 14 recommandés par la norme ASTM E399 [7]. Les critères relaxés sont :

$$
B \geq\left[\frac{K_{\mathrm{Q}}^{2}}{\sigma_{y}^{2}}\right] \quad\left(\text { ASTM E399: } B \geq 2,5\left[\frac{K_{\mathrm{Q}}^{2}}{\sigma_{y}^{2}}\right]\right)
$$

$$
0,8 K_{\mathrm{Q}} \geq K_{\max } \quad\left(\mathrm{ASTM} \mathrm{E} 399: 0,6 K_{\mathrm{Q}} \geq K_{\max }\right)
$$

$$
1,15 \geq\left[\frac{P_{\max }}{P_{\mathrm{Q}}}\right] \quad\left(\text { ASTM E399 }: 1,1 \geq\left[\frac{P_{\max }}{P_{\mathrm{Q}}}\right]\right)
$$

où :

$K_{\max } \quad$ l'intensité maximale de contrainte de cycle de fatigue

$\sigma_{y} \quad$ la limite d'élasticité du rechargement

$B \quad$ l'épaisseur de l'éprouvette CT

$P_{\max } \quad$ la charge maximale appliquée.

Ces nouvelles conditions de fissuration sont considérées comme exprimant valablement le facteur critique d'intensité de contrainte. L'examen des trois équations modifiées montre que la faible ductilité du rechargement ainsi que sa forte hétérogénéité sont à l'origine de cette relaxation.

- les résultats de vitesse de fissuration en fatigue sont conformes aux recommandations de la norme et la progression de la fissure, soumise à une variation cyclique $\Delta K$, vérifie la loi semi-empirique de Paris [8];
- la température variant dans une gamme allant de l'ambiante à $316^{\circ} \mathrm{C}$ n'influence pas la ténacité et ce pour les divers rechargements testés;

- la ténacité des rechargements base-nickel augmente avec la dimension et la fraction volumique du réseau dendritique en phase-nickel ;

- la dureté volumique des rechargements base-nickel est inversement proportionnelle à leur ténacité. Ceci montre la difficulté de développer un rechargement aussi dur que tenace;

- l'addition d'éléments d'alliage stabilisants entraîne une augmentation de la limite élastique et de l'énergie à rupture des dendrites conduisant à un accroissement de la ténacité des rechargements;

- le durcissement par précipitation des phases-nickel dendritiques accroît la ténacité des rechargements.

En tenant compte de ces résultats, Cockerman suggère que la ténacité des rechargements base-nickel est dominée par un mécanisme de pontage de fissure [6]. En effet, la ténacité est modifiée par un changement de la microstructure qui intervient en avant du front de la fissure. L'auteur explique la propagation de fissure par fatigue dans les rechargements base-nickel par la compétition entre deux mécanismes. Le premier, de nature intrinsèque, résulte de la coalescence de cavités qui se forment par rupture d'inclusions ou de gros précipités localisés dans la zone plastique en avant du front de la fissure. Le deuxième, qualifié d'extrinsèque, s'opère dans le sillage du front de la fissure se propageant dans la matrice fragile interdendritique par une action de pontage de fissure dû à l'étirement, la striction progressive et l'allongement plastique de la phase-nickel dendritique. L'interaction de la fissure avec la phase-nickel dendritique peut aussi entraîner un mécanisme de renforcement par courbure et déviation du front de fissure. Ceci est dû aux variations locales de résistance, associées à une faible liaison aux joints de grains ou à la présence de contraintes résiduelles. Le renforcement de la matrice fragile interdendritique par déviation du front de fissure et par pontage de fissure se manifeste par une augmentation substantielle de la ténacité des rechargements. En supposant que ces deux mécanismes de pontage contrôlent la fissuration en fatigue des rechargements base-nickel, la ténacité à rupture peut être décrite par la relation :

$$
\begin{aligned}
K_{\mathrm{tot}} & =\Delta K_{\mathrm{PF}}+\Delta K_{\mathrm{DF}} \\
& =E_{\mathrm{d}} \sqrt{a_{\mathrm{o}} \chi V_{\mathrm{f}}\left(\frac{\sigma_{\mathrm{o}}}{E_{\mathrm{d}}}\right)}+\frac{K_{\mathrm{m}}}{D\left(\cos ^{2} \frac{\theta}{2}\right)+\frac{S}{D+S}}
\end{aligned}
$$

où :

$\Delta K_{\mathrm{PF}} \quad$ la ténacité due au renforcement par pontage de fissure

$\Delta K_{\mathrm{DF}} \quad$ la ténacité due au renforcement par courbure et déviation de fissure

$E_{\mathrm{d}} \quad$ le module élastique de la phase ductile (phasenickel dendritique)

$a_{\mathrm{o}} \quad$ le rayon de la phase ductile 


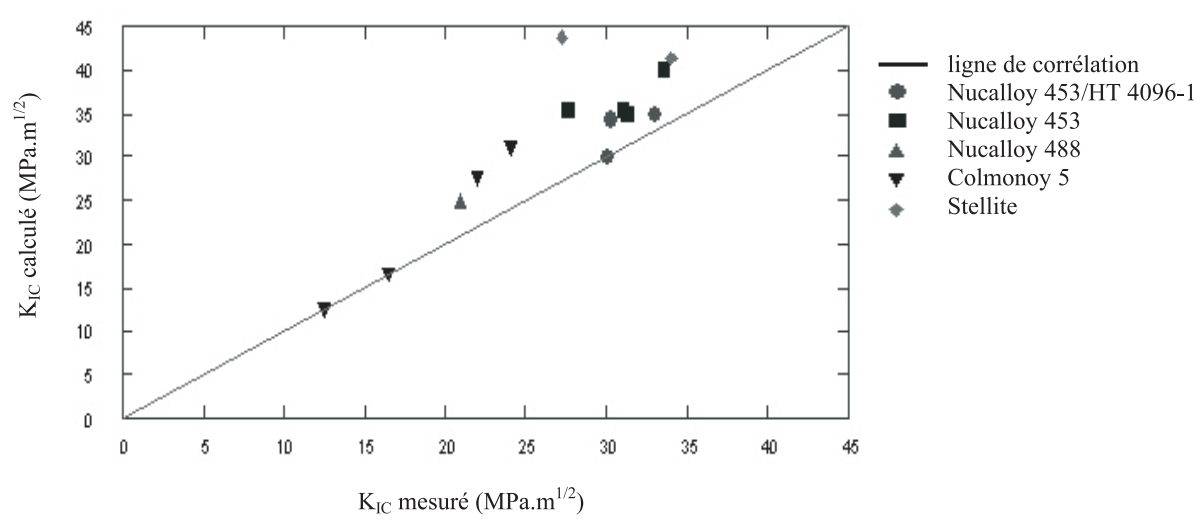

Fig. 1. Corrélation entre les valeurs des facteurs critiques d'intensité de contraintes $K_{\mathrm{IC}}$ calculées et celles mesurées pour plusieurs nuances de rechargements [6].
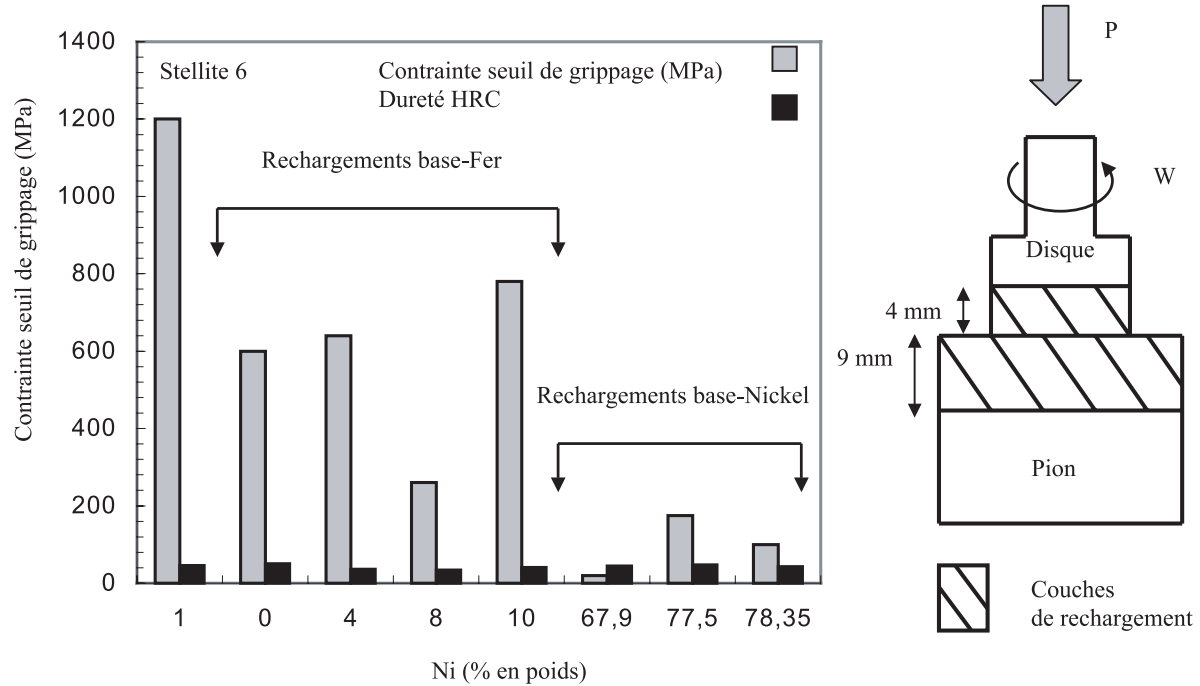

Fig. 2. Variations de la résistance au grippage de plusieurs nuances de rechargements en base-nickel, base-cobalt et base-fer en fonction du pourcentage de nickel [9].

$\chi \quad$ le travail pour la rupture de la phase ductile

$V_{\mathrm{f}} \quad$ la fraction volumique de la phase ductile

$\sigma_{\mathrm{o}} \quad$ la limite élastique de la phase ductile

$K_{\mathrm{m}} \quad$ la ténacité de la matrice fragile interdendritique

$D \quad$ la longueur du défaut

$\theta \quad$ l'angle de déviation

$S \quad$ la distance séparant le défaut de la fissure.

Cette relation donne une bonne corrélation avec les résultats expérimentaux (Fig. 1). En conclusion, les principaux faits expérimentaux de cette étude ainsi que la tentative d'interprétation théorique avancée montrent que les effets de la structure peuvent être décisifs sur l'usinabilité des rechargements base-nickel.

Dans un autre article, Cockerman et al. [9] relatent un certain nombre d'expériences faites suivant la norme de l'ASTM G98 [10] et portant sur la résistance au grippage de plusieurs nuances de rechargements en base-nickel, base-cobalt et base-fer. Ces essais ont été réalisés sous une charge compressive constante, sur des échantillons rechargés, dans une configuration d'essai pion stationnaire sur disque rotatif et à sec (Fig. 2). Les tests ont été maintenus jusqu'à apparition de dommage superficiel résultant $\mathrm{du}$ frottement interfacial pour différentes charges appliquées. Le seuil de contrainte de grippage a été estimé comme la valeur moyenne entre la contrainte maximale de non-grippage et celle minimale de grippage. Les résultats de cette étude (Fig. 2) montrent que la composition chimique, particulièrement le pourcentage en $\mathrm{Ni}$, influe considérablement sur la résistance au grippage. Les rechargements base-nickel présentent une faible résistance au grippage (seuil de contrainte de grippage faible) qui se dégrade d'autant plus que la quantité en Ni augmente. Les rechargements base-fer ont une résistance au grippage élevée en comparaison de ceux à base-nickel mais qui a tendance à diminuer avec une forte addition de $\mathrm{Ni}$. Pour les alliages base-Cobalt aucune propension au grippage n'a été observée et ce pour des pressions de contact allant jusqu'à $1200 \mathrm{MPa}$. Dans les trois types de rechargement, le niveau de dureté est à peu près le même alors que le comportement au grippage diffère. On voit bien ici que la dureté n'est pas un paramètre pertinent pour 


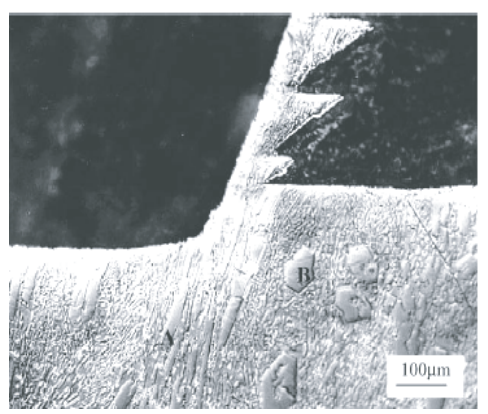

(a)
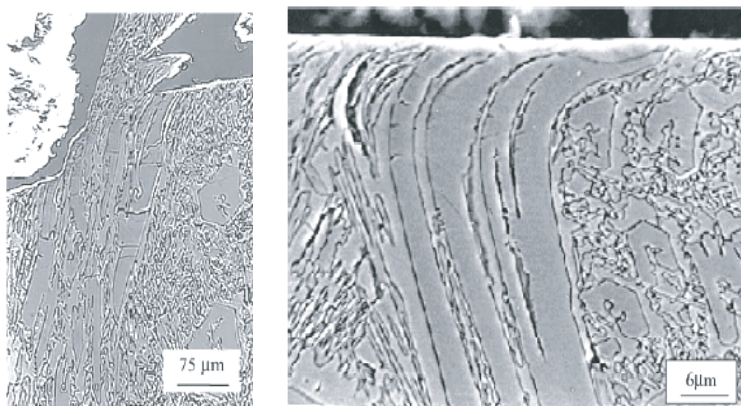

(b)

Fig. 3. Micrographies de coupe interrompue : (a) rupture des inclusions en avant de l'arête tranchante observée en coupe brusquement interrompue. (b) Déformations des carbures primaires en subsurface transitoire [11].

caractériser le comportement à la coupe de ces matériaux. Par ailleurs, pour des structures fortement hétérogènes, tels que ces rechargements réfractaires, la dureté effective à prendre en considération devient une fonction particulière des contributions dues à la dureté intrinsèque de la matrice de l'alliage et de celles des autres phases. Les mécanismes principaux qui gouvernent la faible résistance au grippage des rechargements base-nickel tels qu'ils sont formulés par Cockerman et al. résultent [9] :

- d'une forte action d'adhésion des phases-nickel dendritiques identifiées comme sites d'initiation de l'usure par grippage;

- d'une action de fissuration avec rupture de la matrice fragile interdendritique gouvernant le détachement de particules d'usure;

- d'une action de labourage des débris d'usure accumulés dans le contact et précipitant la fragmentation de la matrice fragile.

Ces résultats apporteront un éclairage sur les mécanismes d'enlèvement de matière des rechargements base-nickel dans la mesure où le copeau (en permanence renouvelé) reste en contact avec l'outil coupant dans des conditions de vitesse et de pression qui sont celles d'un frottement sec collant (grippage entretenu).

Pour préciser l'influence de larges inclusions de carbures de chrome sur l'usinabilité des rechargements à base de fer, Ren et al. [11] ont étudié les mécanismes de formation de copeaux par des essais de coupe interrompue en tournage avec un outil en nitrure de bore cubique polycristallin $(\mathrm{PCBN})$. Le rechargement, épais de $6 \mathrm{~mm}$, présente une structure grossière où une large portion est composée de carbures primaires (50\% en fraction volumique) avec une mixture d'austénite et de carbures fins. Pendant la période de solidification, les carbures primaires montrent une croissance columnaire avec une section transversale hexagonale. Par examen des coupes micrographiques effectuées après essais de coupe interrompus, les auteurs montrent que l'enlèvement de matière s'opère par une rupture des inclusions en avant de l'arête tranchante (Fig. 3a) combinée à une courbure et fissuration des carbures en subsurface transitoire (surface non usinée) (Fig. 3b). Le copeau ainsi formé présente une forme en dents de scie « copeau dentelé » (Fig. 3a). Sur le plan de l'usure, l'écaillage d'arête tranchante et l'usure en dépouille ont été identifiés comme les modes de dégradation dominant d'outil PCBN. Les auteurs suggèrent que le niveau de sollicitation thermomécanique élevé ainsi que le caractère abrasif des carbures sont à l'origine de ces dommages.

De ces éléments bibliographiques, retenons ici :

- que le paramètre «dureté» ne permet en rien d'apprécier la structure des matériaux fortement hétérogènes et ne peut être pris comme indicateur de l'usinabilité des couches du rechargement par soudage d'outillage ;

- que les mécanismes de fissuration des couches du rechargement base-nickel caractérisés par déviations et pontages de fissures témoignent de leur capacité élevée à durcir par écrouissage. Ceci restreindra certainement leur usinabilité;

- que la propension du rechargement base-nickel au grippage suggère que son usinage par enlèvement de matière serait a priori gouverné par un écrouissage important, accompagné de surcontraintes au niveau de l'outil coupant, plus au moins aggravées par la fatigue et les chocs thermiques, peuvent aboutir à des ruptures du matériau de coupe et à son écaillage;

- qu'il est malaisé d'appréhender l'usinabilité de ces couches du rechargement base-nickel si l'on n'a pas décrit très complètement et sous un éclairage original :

1. en quoi consiste la structure des couches du rechargement;

2. comment cette structure est susceptible de se transformer, de se dégrader, d'interagir avec l'outil coupant, sous l'effet des sollicitations thermomécaniques de coupe. Telles sont les raisons qui ont motivé cette étude.

\section{Essais d'usinabilité}

Les essais effectués dans ce travail, afin de qualifier l'usinabilité du rechargement base-nickel, relèvent des tests accélérés d'usinabilité (T.A.U.). La démarche 


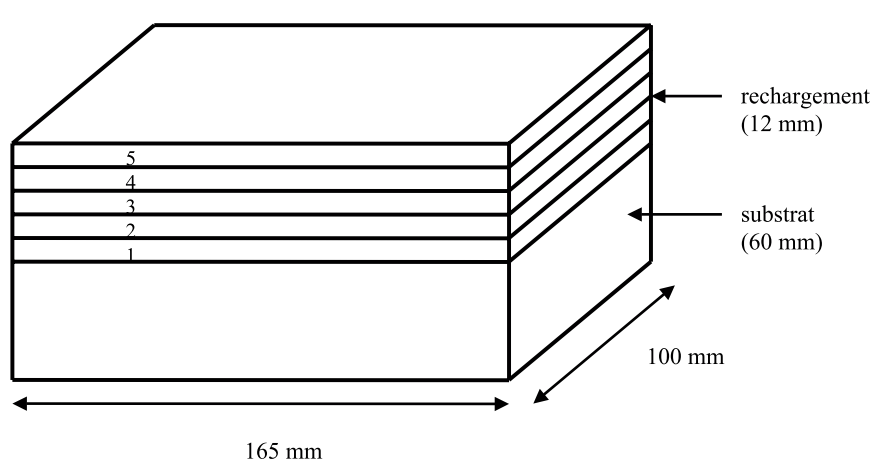

Épaisseur couches avant surfaçage :

couche $\mathrm{n}^{\circ} 1=3 \mathrm{~mm}$ couche $\mathrm{n}^{\circ} 2=3 \mathrm{~mm}$ couche $\mathrm{n}^{\circ} 3=2 \mathrm{~mm}$ couche $\mathrm{n}^{\circ} 4=2 \mathrm{~mm}$ couche $\mathrm{n}^{\circ} 5=2 \mathrm{~mm}$

(a) bloc rechargé

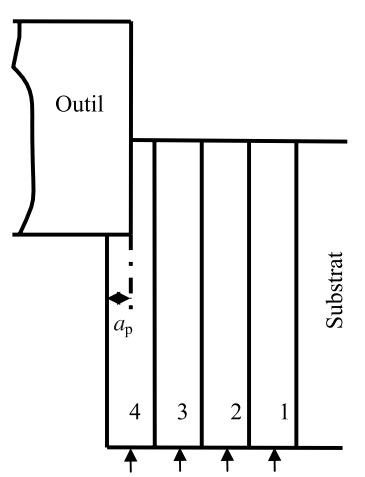

Couches rechargées

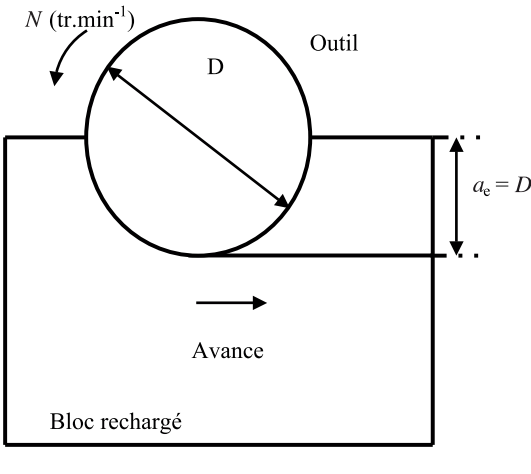

(b) schéma de principe de l'usinage

Fig. 4. Bloc rechargé et schéma de principe de l'usinage.

consiste à tirer partie de la mesure en cours d'essai d'un paramètre physique facilement accessible, dont l'amplitude est corrélée avec l'endommagement de l'outil de coupe par le matériau usiné. Le choix de cette démarche a été motivé par l'obtention d'informations au moindre coût, en un temps court sur prélèvement de matière de petite dimension (essai sur éprouvette). Ci-dessous, une description de la préparation des blocs-éprouvettes ainsi que la conduite de l'essai instrumenté sont données.

\subsection{Préparation des blocs-éprouvettes}

La figure 4a schématise le bloc-éprouvette utilisé pour les tests d'usinabilité accélérés. Il s'agit d'un bloc d'acier $56 \mathrm{NiCrMoV} 7$ épais de $60 \mathrm{~mm}$ et rechargé par soudage au fil fourré (MIG pulsé semi-automatique) avec cinq couches dont l'épaisseur unitaire varie entre 2 et $3 \mathrm{~mm}$. Le rechargement utilisé est de type Hastelloy C, considéré comme le plus représentatif des rechargements préventifs. La composition chimique ainsi que les propriétés mécaniques et physiques du rechargement et du substrat sont reportées sur le tableau 1. Le rechargement brut de soudage présente une surface fortement ondulée avec une variation de planéité allant de 2 à $3 \mathrm{~mm}$. Ces ondulations superficielles entraînent une usure prématurée des outils de coupe due essentiellement aux instabilités de coupe inhérentes aux grandes variations d'épaisseur du copeau. Pour chaque bloc-éprouvette testé, des opérations de surfaçage ont été effectuées préalablement afin d'obtenir une surface plane. Ainsi, sur le brut de soudage, systématiquement la cinquième couche $(2 \mathrm{~mm})$ et une partie de la quatrième couche $(1 \mathrm{~mm})$ ont été enlevées. Une analyse métallographique couplée à un relevé en profil de microdureté a été réalisée sur chaque blocéprouvette avant usinage.

\subsection{Conduite de l'essai de fraisage}

Des essais de rainurage à l'air comprimé, en pleine matière avec des paramètres d'usinage constants (vitesse de coupe $V_{\mathrm{c}}=80 \mathrm{~m} \cdot \mathrm{min}^{-1}$, engagements axial $a_{\mathrm{p}}=0,12 \mathrm{~mm}$ et radial $a_{\mathrm{e}}=12 \mathrm{~mm}$, avance par tour et par dent $f_{z}=0,05 \mathrm{~mm}$.dent ${ }^{-1} \cdot \mathrm{tr}^{-1}$ ) constituent les T.A.U. où seule varie la nature de la couche du rechargement. Ceci dans un objectif de corrélation entre structure du rechargement et usinabilité. La figure 4b montre le schéma de principe des essais de fraisage en pleine matière. Les outils de coupe utilisés sont des fraises à quatre dents $(z=4)$ de diamètre $\Phi=12 \mathrm{~mm}$ en carbure micro-grain revêtu en nitrure de titane aluminium TiAlN additionné d'une couche en bisulfure de molybdène $\mathrm{MoS}_{2}$. Le critère d'usure adopté dans tous les essais est 
Tableau 1. Composition chimique, caractéristiques physiques et mécaniques du substrat et du rechargement.

\begin{tabular}{|c|c|c|c|c|c|}
\hline & $\begin{array}{c}\text { Masse } \\
\text { volumique } \\
\left(10^{3} \mathrm{~kg} \cdot \mathrm{m}^{-3}\right)\end{array}$ & $\begin{array}{c}\text { Conductivité } \\
\text { thermique } \\
\left(\mathrm{W} \cdot \mathrm{m}^{-1} \cdot{ }^{\circ} \mathrm{C}^{-1}\right) \\
\left(20^{\circ}\right)\end{array}$ & 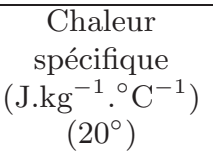 & $\begin{array}{c}\text { Diffusivité } \\
\text { thermique } \\
\left(\mathrm{m}^{2} \cdot \mathrm{s}^{-1}\right) \\
\left(20^{\circ}\right)\end{array}$ & $\begin{array}{c}\text { Module } \\
\text { d'Young } \\
\text { (GPa) }\end{array}$ \\
\hline Hastelloy C & 8,8 & 10,0 & 420 & $2,7 \times 10^{-6}$ & 213 \\
\hline \multicolumn{4}{|c|}{ Mo $15,00-17,00$ Co $0,50 \max \mathrm{Ni}>60,00(\%)$} & \multicolumn{2}{|c|}{$\begin{array}{l}\mathrm{Fe}<2,0 \text { W 3,00-4,50 } \\
0(\%)\end{array}$} \\
\hline $56 \mathrm{NiCrMoV} 7$ & 7,85 & 34,4 & 460 & $9,5 \times 10^{-6}$ & 207 \\
\hline C 0,55 & Si 0,29 Mn 0,70 & Ni 1,70 Mo 0,50 & $\mathrm{~V} 0,10 \mathrm{Cu} 0$ & 23 Fe 94,83 & \\
\hline
\end{tabular}

la défaillance totale de l'outil de coupe selon la norme NF E 66-505 [12]. Tous les essais ont été réalisés sur un centre d'usinage grande vitesse MIKRON HSM 700 (puissance maximale admissible $12 \mathrm{~kW}$, vitesse de rotation maximale 42000 tr.min ${ }^{-1}$, diamètre d'outil maximal $12 \mathrm{~mm}$ ). Pour chaque essai d'usure sont mesurés la puissance, la température ainsi que les efforts de coupe et ce toutes les trois passes $\left(3 \times a_{\mathrm{p}}=0,36 \mathrm{~mm}\right)$, après balayage complète de la surface du bloc, en partant de la couche supérieure du rechargement jusqu'au substrat. En courant triphasé, la mesure de puissance s'effectue par le biais d'un transducteur de puissance monté en série entre les variateurs et le moteur de broche. La puissance absorbée par le processus de coupe est évaluée par la différence entre la puissance à vide $P_{\mathrm{o}}$ et celle en charge $P_{\mathrm{c}}$ (cette dernière mesure est réalisée lorsque l'outil est totalement en prise). La mesure des efforts de coupe en cours d'usinage consiste en une chaîne d'acquisition composée d'un dynamomètre Kistler 9255B et d'un amplificateur de charge Kistler 5019B à trois canaux. L'amplificateur transforme les signaux de charge du dynamomètre en tensions de sortie $( \pm 10$ Volts) proportionnelles aux forces appliquées. La mesure de la température consiste en l'acquisition d'un thermographe à l'aide d'une caméra infrarouge AGEMA (Thermovision $900 \mathrm{LW}$ ) avec un logiciel de visualisation et d'analyse de champs thermiques enregistrés. La température moyenne a été estimée sur une surface usinée de $0,738 \mathrm{~mm}^{2}$ située à la sortie de la fraise.

\section{Résultats et discussions}

\subsection{Analyse métallurgique du rechargement}

La microstructure des couches de rechargement observée au microscope optique après une attaque électrolytique, à l'acide chromique $\mathrm{H}_{2} \mathrm{CrO}_{3}$ en solution à $10 \%$, a montré une forte hétérogénéité (Fig. 5). Celleci consiste en un réseau dendritique composé d'une phase nickel et séparé par des phases interdendritiques, ainsi que des particules métalliques non fondues. Un relevé de microdureté en profil le long des couches de rechargement a été effectué (Fig. 6). Les mesures consistent en des indentations Vickers sous une charge constante de $30 \mathrm{~g}$.
Comme le montre la figure 6, à l'exception de l'interface entre la première couche et le substrat, la microdureté mesurée en profil est homogène et a pour valeur moyenne $230 \mathrm{HV}$ environ. Ceci confirme que la microdureté n'est pas un paramètre révélateur de l'hétérogénéité de la microstructure.

\subsection{Analyse des résultats des T.U.A.}

Le rainurage pleine matière des quatre couches de rechargement, selon le critère d'usure retenu dans cette étude, a nécessité l'utilisation de quatre outils. La figure 7 regroupe l'évolution de la dureté superficielle après usinage. Les mesures de dureté Vickers ont été effectuées sous une charge de $30 \mathrm{~kg}$. Après la première passe de rainurage dans la $4^{\mathrm{e}}$ couche, la dureté superficielle du matériau est de $247 \mathrm{HV}$. Ensuite la dureté augmente régulièrement après usinage jusqu'à la défaillance brutale de l'outil de coupe (durée de vie de l'outil $\left.\mathrm{n}^{\mathrm{o}} 1: T_{1}=12,8 \mathrm{~min}\right)$ où la dureté s'élève à 296 HV. Ces résultats mettent clairement en évidence l'effet d'écrouissage superficiel par consolidation plastique. En effet, aux faibles avances (dans ces essais : $f_{z}=$ $\left.0,05 \mathrm{~mm} \cdot \mathrm{tr}^{-1}\right)$, l'écrouissage dû à la déformation plastique est prédominant sur l'adoucissement thermique qui se traduit plutôt par une transformation de phase [13]. Le prolongement des essais avec un outil neuf sur la troisième couche est accompagné d'un retour de la dureté jusqu'à sa valeur initiale. Ceci signifie que l'écrouissage de la $4^{\text {e }}$ couche en terme d'augmentation de dureté est influencé par l'usure de l'outil de coupe. Ce durcissement superficiel progressif est observé sur les $3^{\mathrm{e}}$ et $2^{\mathrm{e}}$ couche accompagné par des durées de vie faibles des outils $n^{\circ} 2$ et $n^{\circ} 3$ respectivement $T_{2}=17,5 \mathrm{~min}$ et $T_{3}=12,8 \mathrm{~min}$. Cependant, le fraisage de la première couche s'effectue sans que le critère de défaillance brutale de l'outil ne soit atteint. La dureté n'évolue pas de manière significative, mis à part dans la zone thermiquement affectée (zone de transformation martensitique). Ce résultat montre que la $1^{\mathrm{e}}$ couche a une composition chimique différente des autres couches en raison de la dilution importante de fer du substrat vers le rechargement pendant le processus de soudage. Ceci est d'autant vrai que le taux de dilution propre au procédé 


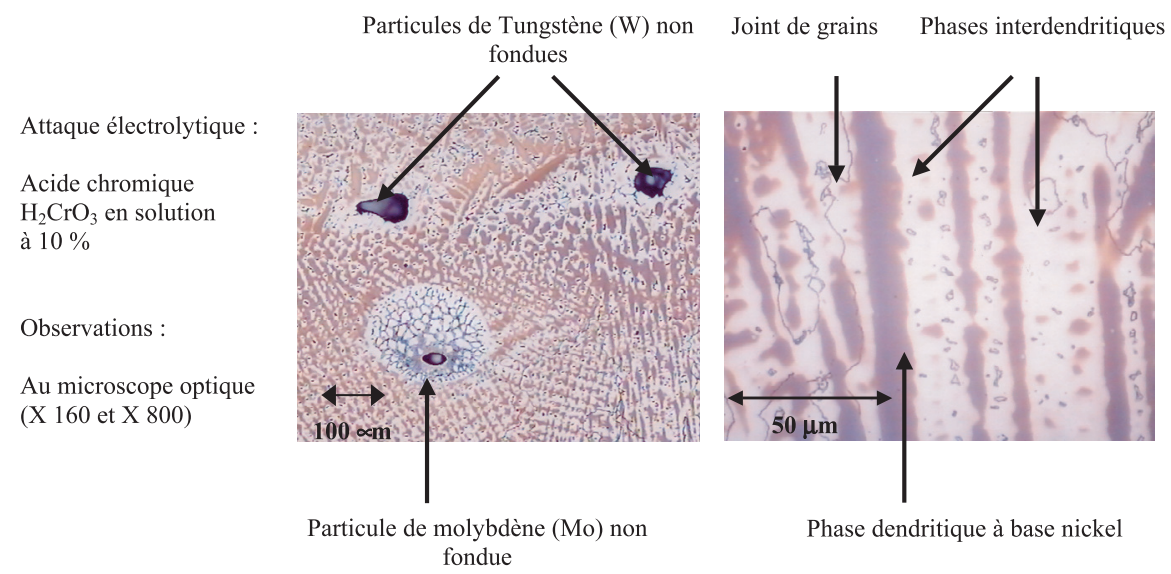

Fig. 5. Microstructure en profil du rechargement en superalliage à base-nickel.

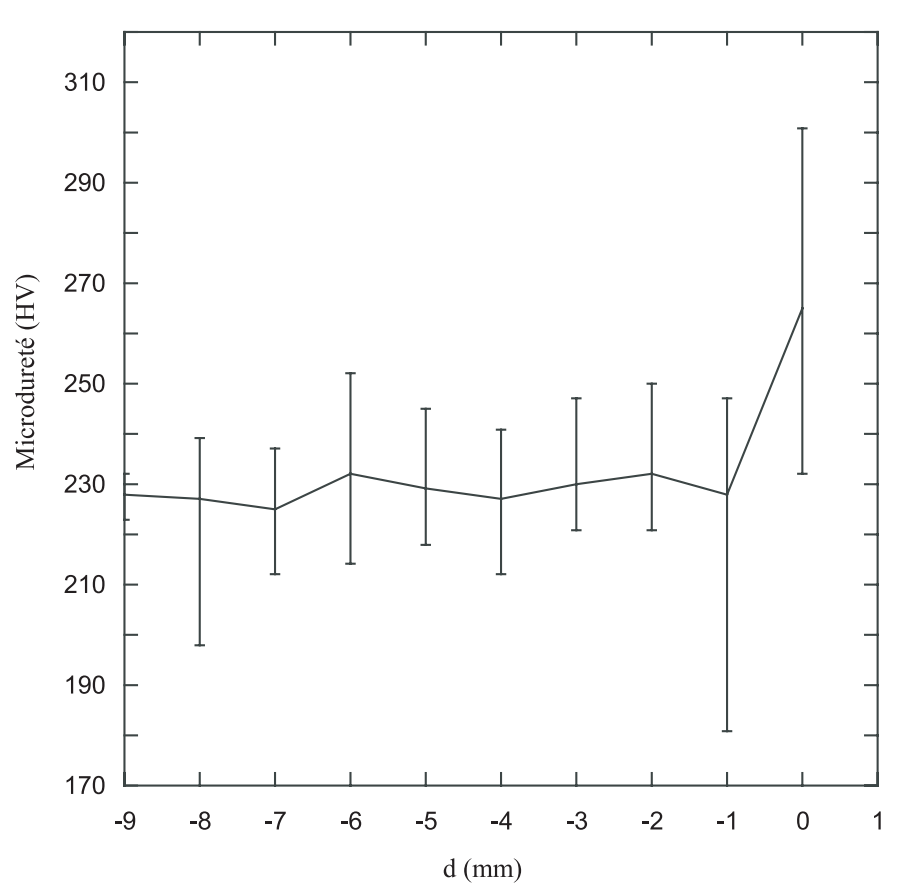

Fig. 6. Mesures de microdureté en profil effectuée sur les couches de rechargement.

de soudage utilisé (MIG) est très élevé de l'ordre de 15 à $30 \%$ [1]. L'évolution de la dureté superficielle en fonction de l'épaisseur usinée du rechargement (Fig. 7) met aussi en évidence l'influence de la cinétique d'endommagement des outils, en coupe discontinue, sur l'écrouissage des couches supérieures. Il y a lieu de constater également la différence d'usinabilité entre la première couche attenante au substrat et les couches supérieures.

\subsection{Analyse énergétique}

En coupe discontinue, l'énergie absorbée par la formation d'un volume de copeau prend en considération l'ensemble des énergies [14]:

- l'énergie dissipée par frottement entre l'outil et la pièce et entre l'outil et le copeau;
- l'énergie absorbée par la déformation élastique et plastique lors de la formation du copeau;

- l'énergie absorbée par les chocs d'entrée de l'outil dans la pièce.

La figure 8 représente la variation de l'énergie spécifique de coupe (rapport de la puissance de coupe au débit de matière enlevée) en fonction de l'épaisseur du dépôt usiné. Cette variation est similaire à celle observée pour l'évolution de la dureté superficielle (Fig. 7). Ainsi, chaque prolongement d'essai avec un outil neuf est marqué par une chute de l'énergie jusqu'à sa valeur initiale $\left(70 \times 10^{8}{\left.\mathrm{~J} . \mathrm{m}^{-3}\right)}^{-3}\right.$. Cependant, une usure croissante pour un même outil se traduit par une augmentation de l'énergie spécifique de coupe. Cette augmentation fait apparaître un écrouissage superficiel qui, au cours du processus de coupe, élève la dureté du rechargement. En effet, la perte progressive d'acuité d'arête fait transiter la coupe d'enlèvement de matière par cisaillement (formation du copeau) à un labourage (bourrelet frontal) et écrouissage par déformation plastique. Cette transition se trouve confirmée par l'augmentation très significative de l'effort de coupe axial (Fig. 9) et un échauffement de la couche usinée (Fig. 10) qui est d'autant plus élevé que la conductivité thermique du rechargement est très faible (Tab. 1). Sur la figure 10 est en effet rapportée l'évolution de la température moyenne relevée sur la surface de la pièce à la sortie immédiate de la fraise. Si, à présent, on se réfère à la température moyenne sur la face de coupe d'outil telle qu'elle a été évaluée par Shaw [15], celleci varie selon l'équation (5). Cette relation dérive d'une analyse dimensionnelle basée sur la réponse thermophysique du matériau usiné et les phénomènes thermiques du frottement lors de la coupe et proposée initialement par Kronenberg, puis modifiée par Shaw [15]

$$
\frac{\bar{\theta}_{\mathrm{T}}}{u_{\mathrm{c}}}\left[\frac{K \rho C}{V_{\mathrm{c}} f_{z}}\right]^{\frac{1}{2}}=\text { const. }
$$

où :

$\bar{\theta}_{\mathrm{T}} \quad$ la température moyenne sur la face de coupe d'outil

$u_{\mathrm{c}} \quad$ l'énergie spécifique de coupe

$V_{\mathrm{c}} \quad$ la vitesse de coupe 


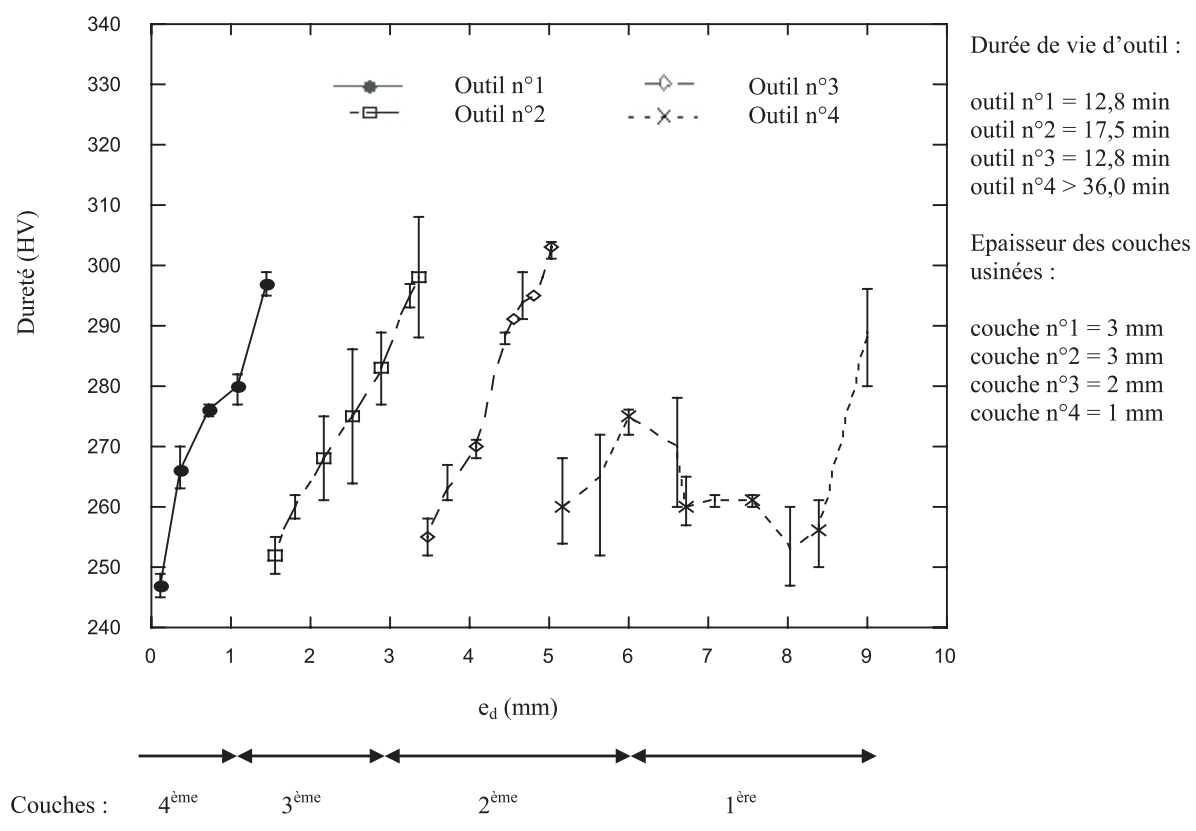

Fig. 7. Évolution de la dureté en surface après usinage en fonction de l'épaisseur du dépôt usiné $\left(V_{\mathrm{c}}=80 \mathrm{~m} \cdot \mathrm{min}^{-1}, f_{z}=\right.$ $0,05 \mathrm{~mm}, a_{\mathrm{e}}=12 \mathrm{~mm}, a_{\mathrm{p}}=0,12 \mathrm{~mm}, D=12 \mathrm{~mm}, z=4$ dents).

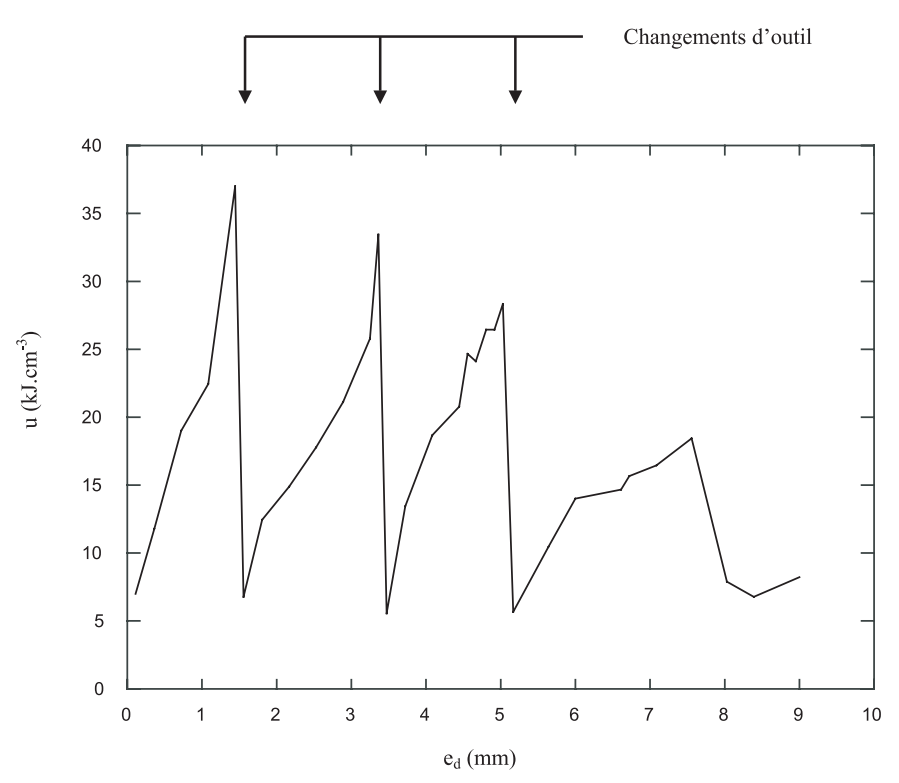

Fig. 8. Évolution de l'énergie spécifique de coupe en fonction de l'épaisseur du dépôt usiné $\left(V_{\mathrm{c}}=80 \mathrm{~m} \cdot \mathrm{min}^{-1}, f_{z}=\right.$ $0,05 \mathrm{~mm}, a_{\mathrm{e}}=12 \mathrm{~mm}, a_{\mathrm{p}}=0,12 \mathrm{~mm}, D=12 \mathrm{~mm}$, $z=4$ dents).

$f_{z} \quad$ l'épaisseur du copeau non déformé (avance par dent et par tour)

$C$ la capacité thermique massique du matériau usiné

$K \quad$ la conductivité thermique du matériau usiné

$\rho \quad$ la densité massique du matériau usiné.

Dans cette formule, on note que, des différents paramètres d'usinage, la température d'outil est plus sensible à la variable vitesse de coupe ainsi qu'à l'avance

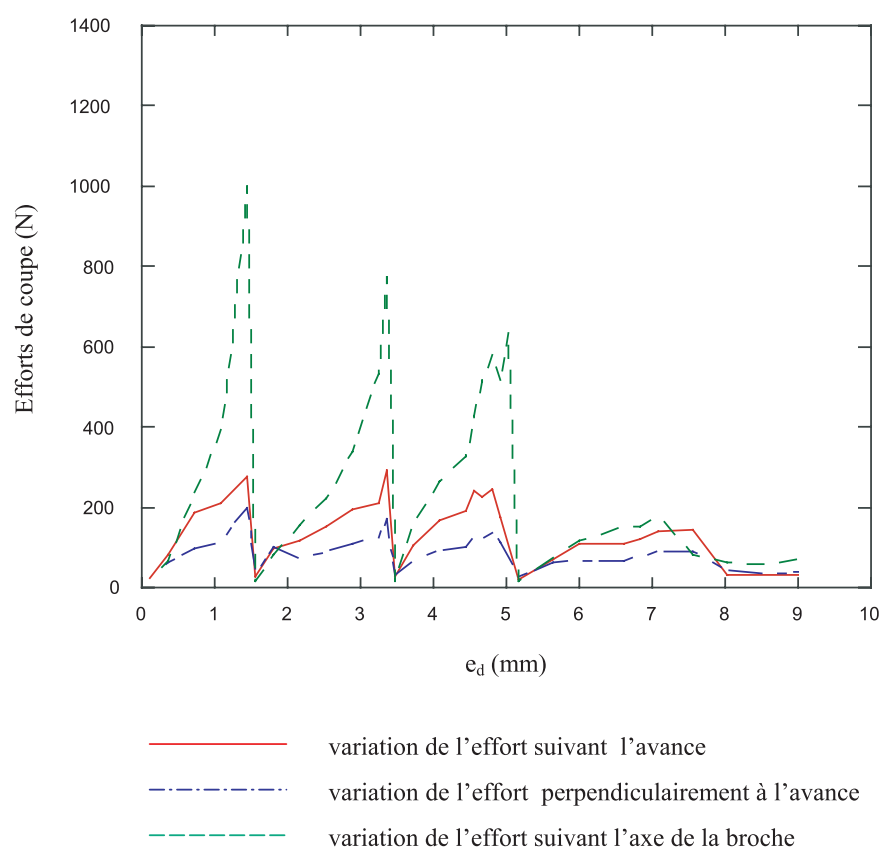

Fig. 9. Évolution des efforts de coupe avec l'épaisseur du dépôt usiné $\left(V_{\mathrm{c}}=80 \mathrm{~m} \cdot \mathrm{min}^{-1}, f_{z}=0,05 \mathrm{~mm}, a_{\mathrm{e}}=12 \mathrm{~mm}\right.$, $a_{\mathrm{p}}=0,12 \mathrm{~mm}, D=12 \mathrm{~mm}, z=4$ dents).

dans la matière. Ces deux variables interviennent avec un même niveau d'influence (à la puissance $1 / 2$ ). Il n'en est pas de même du facteur $\sqrt{K \rho C}$, qualité spécifique du matériau usiné, et la formule montre la loi suivante.

Lors de la coupe, la température d'outil sera d'autant plus basse que le facteur $\sqrt{K \rho C}$ du matériau usiné sera plus élevé. En effet, lorsqu'un outil usine un matériau à conductibilité thermique élevée, c'est le copeau, 


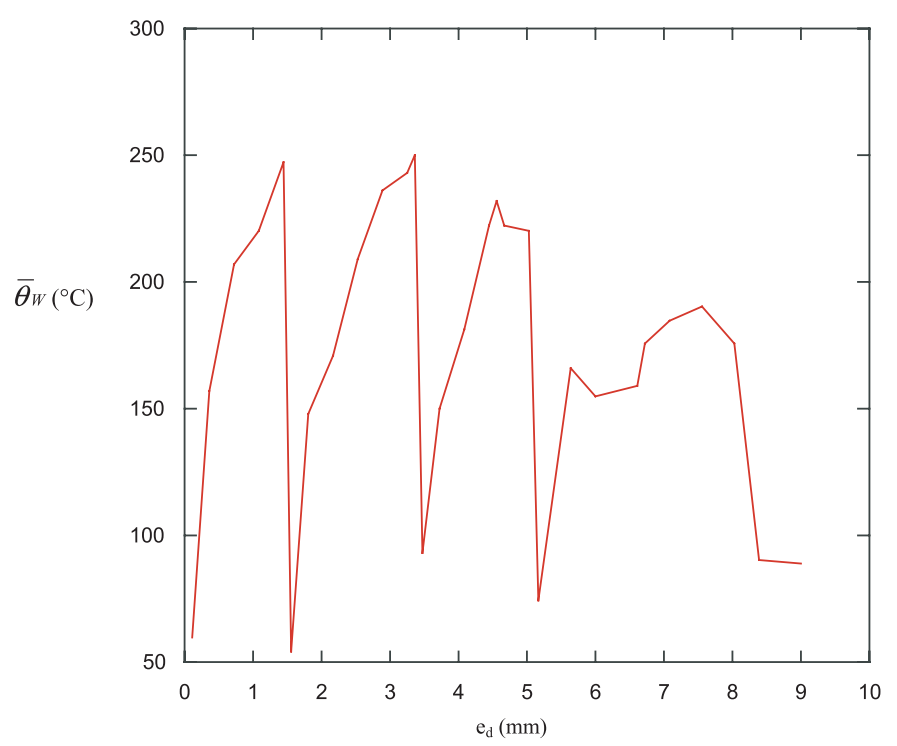

Fig. 10. Évolution de la température relevée sur la surface de la pièce à la sortie de la fraise avec l'épaisseur du dépôt usiné $\left(V_{\mathrm{c}}=80 \mathrm{~m} \cdot \mathrm{min}^{-1}, f_{z}=0,05 \mathrm{~mm}, a_{\mathrm{e}}=12 \mathrm{~mm}, a_{\mathrm{p}}=0,12 \mathrm{~mm}\right.$, $D=12 \mathrm{~mm}, z=4$ dents).

circonstance très favorable qui emmagasine plus de chaleur que l'outil. Toutefois, deux cas peuvent advenir :

- s'il y a contact prolongé du copeau sur l'outil, celui-ci a le temps de recevoir de la chaleur de celui-là;

- s'il n'y a qu'un contact bref, l'outil ne s'échauffera pas.

Toujours dans la formule (5), on remarque que dans la variation de la température d'outil l'énergie spécifique de coupe est affectée de l'exposant 1. Ceci montre que la température est aussi sensible à cette variable que les paramètres de coupe (vitesse de coupe et avance). Or si la variable énergie spécifique de coupe, grandeur homogène à une contrainte, est, en première approximation, indépendante de la vitesse de coupe; elle est, cependant, fonction décroissante de l'épaisseur de copeau non déformée ou avance $f_{z}$. Cette décroissance en fonction de l'avance traduit un effet de taille (size effect) qui s'exprime aux faibles avances par [15] :

$$
u_{\mathrm{c}} \approx \frac{1}{f_{z}^{0,2}}
$$

De sorte que la relation (5) s'écrit :

$$
\bar{\theta}_{\mathrm{T}} \approx(K \rho C)^{-\frac{1}{2}} V_{\mathrm{c}}^{\frac{1}{2}} f_{z}^{0,3}
$$

L'examen de la figure 10 montre que l'évolution des températures moyennes à la surface des couches usinées est fonction croissante de l'épaisseur enlevée. Il montre aussi que les températures maximales atteintes dans les trois couches supérieures varient entre $225^{\circ} \mathrm{C}$ et $250{ }^{\circ} \mathrm{C}$. Ainsi, pour le triplet outil-matière-environnement étudié, appelons $\bar{\theta}_{\mathrm{W}}$ la température moyenne à la surface usinée; admettant aussi l'hypothèse assignant que les quantités de chaleur se répartissent comme si l'interface était une source émettrice et si les surfaces balayées recevaient un flux, ceci nous amène au résultat limite que voici :

$$
\bar{\theta}_{\mathrm{W}} \approx V_{\mathrm{c}} f_{z}^{0,6}
$$

Dans les conditions de coupe utilisées, le coefficient $V_{\mathrm{c}} f_{z}^{0,6}=220,964$. Il est clair que cette réflexion, même très approximative, montre que l'outil sera soumis à des températures beaucoup plus élevées et prévoit que le ou les modes d'usure d'outil dominants seront d'origine thermique (relation (7)). Ceci est en grande partie dû̀ à la faible conductivité thermique du rechargement basenickel. En effet, à conditions de coupe identiques, la relation (5) suggère que pour différents matériaux usinés avec le même outil, la vitesse de coupe qui donnerait la même température serait :

$$
V_{\mathrm{c}} \approx \frac{K \rho C}{u_{\mathrm{c}}^{2}}
$$

Le tableau 2 donne les valeurs du facteur spécifique $K \rho C$ ainsi que l'énergie spécifique de coupe $u_{\mathrm{c}}$ pour l'acier d'outillage rechargé, pris comme matériau à usinabilité de référence, et le rechargement base-nickel comparées à celles du rechargement base-cobalt et deux matériaux d'usage aéronautique; puis une estimation du rapport de vitesse de coupe :

$$
\frac{V_{\mathrm{c}}}{V_{\mathrm{c}_{\text {acier }}}}=\frac{K \rho C}{(K \rho C)_{\text {acier }}}\left(\frac{u_{\mathrm{c}_{\text {acier }}}}{u_{\mathrm{c}}}\right)^{2}
$$

Ce rapport quantifiera, en première approximation, l'usinabilité des couches du rechargement base-nickel relative à celle d'acier d'outillage $55 \mathrm{NiCrMoV} 7$ (substrat rechargé) en terme de vitesse de coupe permise. Il donnera aussi un sens du partage physique des calories lors de la coupe tel qu'il est formulé dans la relation (5). Ainsi à l'examen du tableau 2, il est clair que l'alliage d'aluminium $7175 \mathrm{~T} 73$ peut être usiné à très grande vitesse par rapport à l'acier d'outillage $55 \mathrm{NiCrMoV} 7$ alors que les couches rechargées en superalliage réfractaire base-nickel doivent être usinées à des vitesses largement plus faibles. Cette comparaison préjuge aussi une usinabilité améliorée des rechargements base-cobalt par rapport à ceux en basenickel. Elle confirme par ailleurs que la vitesse de coupe permise est une fonction croissante du facteur $\frac{K \rho C}{u_{\mathrm{c}}^{2}}:$ l'usinage à grande vitesse n'est économiquement viable que lorsque la valeur de ce facteur est très élevée.

\subsection{Analyse de copeau}

Pour tous les essais, la forme micrographique du copeau résultante des essais de rainurage est celle d'un copeau dentelé et ce quelque soit la couche usinée (Fig. 11). Ce type de copeau est formé d'éléments séparés, dus plutôt à une rupture du matériau qu'à un cisaillement de celui-ci (Fig. 11b). Toutefois, les interprétations avancées pour expliciter les mécanismes de formation du copeau dentelé ne sont pas unanimes. Elles se classent globalement selon deux thèses. La première s'appuie entre autres 

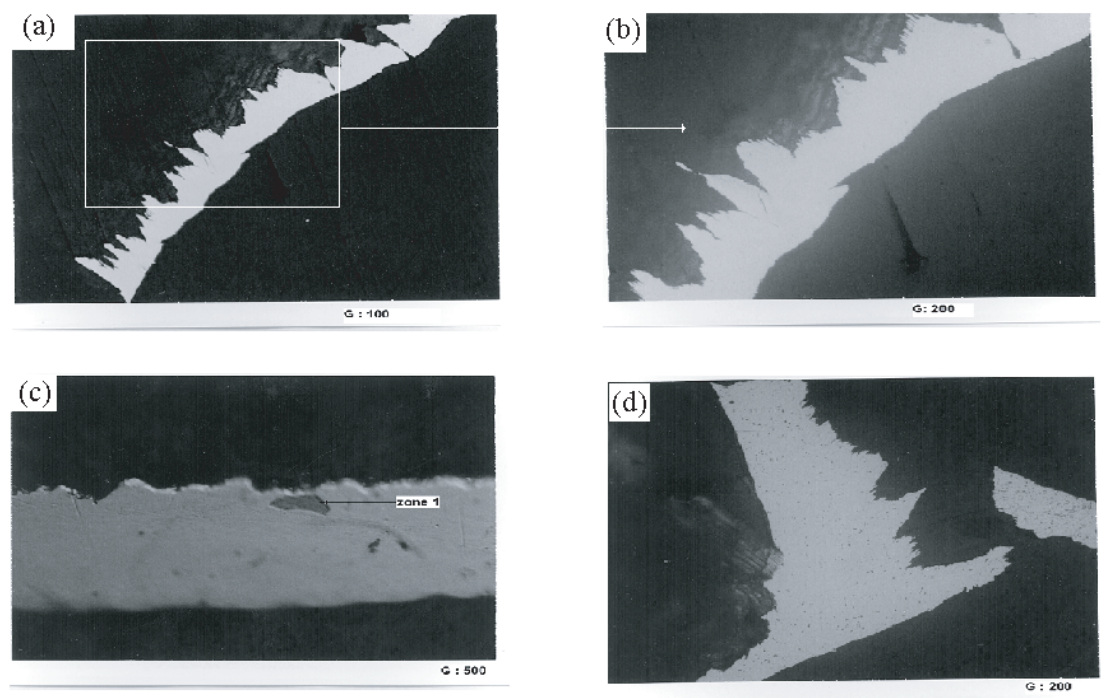

Fig. 11. Sections métallographiques des copeaux en rechargement base-nickel : (a) section longitudinale du copeau montrant qu'il est formé d'éléments séparés, dus plutôt à une rupture du matériau qu'à un cisaillement. (b) Vue grossie de la forme du copeau confirmant l'existence de rupture. (c) Gros précipité composant le copeau. (d) Multiples points noirs correspondant aux carbures de chrome comme confirmé par l'attaque chimique de Murakami.

Tableau 2. Vitesse de coupe relative à une même température d'outil pour différents matériaux usinés.

\begin{tabular}{cccc}
\hline Matériaux usinés & $u_{\mathrm{c}} \mathrm{J} \cdot \mathrm{m}^{-3}\left(10^{8}\right)$ & $\frac{K \rho C}{u_{\mathrm{c}}^{2}} \mathrm{~m}^{2} \cdot \mathrm{s}^{-1} \cdot{ }^{\circ} \mathrm{C}^{-2}\left(10^{-12}\right)$ & $\frac{V_{\mathrm{c}}}{V_{\mathrm{c}_{\text {acier }}}}=\frac{K \rho C}{(K \rho C)_{\text {acier }}}\left(\frac{u_{\mathrm{c} \text { acier }}}{u_{\mathrm{c}}}\right)^{2}$ \\
\hline $\begin{array}{c}\text { Acier d'outillage : } \\
\text { 56NiCrMoV7 }\end{array}$ & 25,06 & 19,72 & 1 \\
$\begin{array}{c}\text { Alliages base-nickel : } \\
\text { Rechargement Hastelloy C }\end{array}$ & 75 & 0,66 & 0,03 \\
Alliage de titane : \\
$\begin{array}{c}\text { TA6V } \\
\text { Alliage d'aluminium : } \\
\text { 7175T73 }\end{array}$
\end{tabular}

sur les travaux de Komanduri [16,17] qui attribue l'origine du copeau dentelé ou copeau à zone de cisaillement localisée à une instabilité plastique. Cette dernière se manifeste lors de l'usinage en deux phases; une d'aplatissement de la matière usinée devant l'outil où la déformation est très localisée dans la zone de cisaillement primaire ; l'autre d'instabilité plastique à l'origine d'un cisaillement qualifié de catastrophique. Ce dernier point est contesté par les partisans de la deuxième thèse tels que Schultz [18] et Shaw [15] qui attribuent plus d'importance au frottement outil/copeau (zone de cisaillement secondaire). L'examen de la figure 11 (Figs. 11c et 11d) met en évidence, dans le cas d'usinage des couches base-nickel, cette importance : le copeau est chargé de précipités et de points noirs caractéristiques de carbures de chrome. Ces phases très abrasives favoriseront un frottement intense au niveau du contact outil/copeau. La formation de copeau dentelé dans ce cas correspond ainsi à un processus intermittent caractérisé par une fissuration puis un cisaillement sur la surface de séparation du copeau et de la pièce usinée. L'importance du frottement dans la zone de cisaillement secondaire (face de coupe/copeau) peut être appréciée également en estimant la vitesse de glissement du copeau par rapport à l'outil. Celle-ci a été établie par Shaw, en coupe orthogonale et pour un matériau à comportement thermoviscoplastique rigide, comme suit [15] :

$$
\bar{V}_{\mathrm{g}}=r V_{\mathrm{c}}
$$

où $r=\frac{f_{z}}{h_{\mathrm{c}}}$ représente le rapport de compression exprimant le ratio entre l'épaisseur du copeau non déformée et l'épaisseur moyenne du copeau formé $h_{\mathrm{c}}$. Le rapport moyen de compression calculé pour les essais de coupe effectués équivaut à $r=0,78$ (Fig. 12). Habituellement, ce niveau de rapport de compression, lors de l'usinage des matériaux ductiles indique une bonne usinabilité. Ceci 


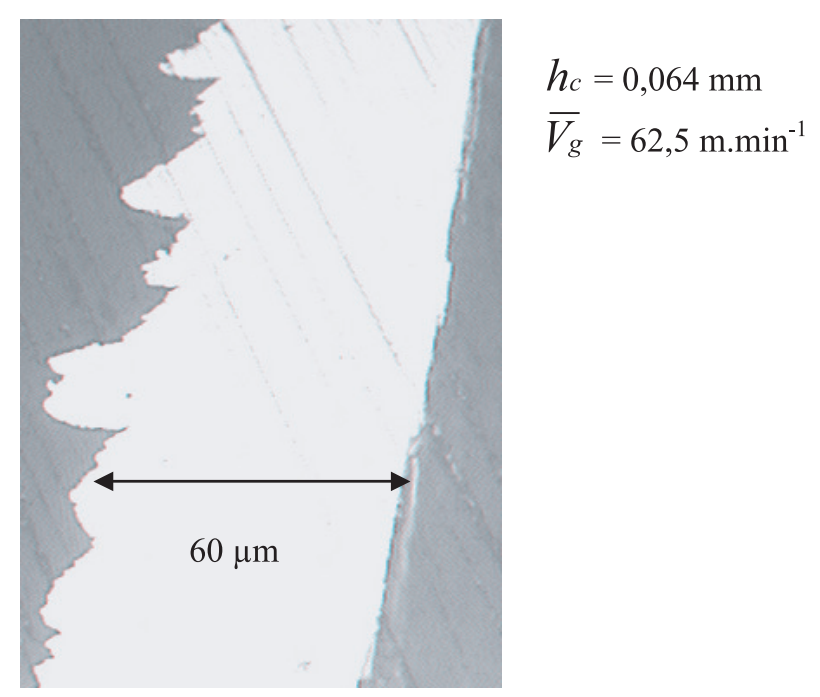

Fig. 12. Estimation de l'épaisseur moyenne du copeau en dents de scie formé lors des essais de rainurage des couches de rechargement base-nickel.

n'est pas le cas du rechargement étudié dans ce travail. Le même constat a été observé par Ren et al. [11] lors de l'usinage des rechargements base fer à forte inclusion de carbures. Le fait que le rapport de compression ne constitue pas un bon indicateur peut s'expliquer par la nature très hétérogène du rechargement ainsi que l'effet des facteurs cinématique et géométrique liés à la présente configuration de coupe « fraisage pleine matière ». Néanmoins, il est utile de calculer la vitesse de glissement de copeau qui est de l'ordre de $62,5 \mathrm{~m} . \mathrm{min}^{-1}$; soit $78 \%$ de la vitesse de coupe. Ceci laisse entendre des conditions sévères du chargement tangentiel auxquelles seront soumises l'arête tranchante ainsi que la face de coupe frottant contre un copeau abrasif (phases carbures et précipités).

\subsection{Endommagements des outils de coupe}

La figure 13 montre la photographie d'un outil neuf sur lequel sont indiquées les zones d'observation analysées après toutes les séries de trois passes axiales d'usinage $\left(3 \times a_{\mathrm{p}}=0,36 \mathrm{~mm}\right)$. Étant donnée la faible profondeur de passe axiale, les endommagements (entailles et écaillages) sont essentiellement localisés sur la pointe d'outil (Fig. 14). On note aussi une cratérisation de la face de coupe ainsi qu'un collage irrégulier des copeaux sur l'arête de coupe (Fig. 14 - zone 1 et zone 2). L'usure en dépouille est plus significative que la cratérisation et se traduit par une rupture de la lèvre d'arête (Fig. 14 - zone 2). Ces dommages (écaillement d'arête, copeau adhérent et effondrement d'arête) témoignent de la sévérité des conditions de coupe en terme de température et de pression. Ils laissent penser aussi que le mécanisme d'usure dominant est de nature adhésif. En effet, le mauvais écoulement du copeau conduit à son collage sur l'arête. Ce copeau adhérent, partiellement évacué et en permanence renouvelé, provoque l'apparition de fissures et d'ébréchures sur l'arête (écaillage d'arête). Ce résultat corrobore la discussion développée dans la section 4.3 sur la possible prédominance des modes d'usure d'origine thermique. Par ailleurs, Ezugwu et al. [2] avancent que, d'une façon générale, l'adhésion des copeaux sur les outils au cours de l'usinage des alliages à base-nickel, sous forme de matériaux massifs, provoque le détachement de particules du matériau de coupe sous forme d'entailles. La tendance à l'adhésion de ces alliages est attribuée à la faible résistance au grippage de la phase nickel, confirmée par les travaux de Cockerman et al. [9]. L'effondrement de l'arête tranchante (côté face de dépouille) avec la perte de matière peut être attribué au caractère abrasif de la microstructure de l'alliage (usure abrasive).

\section{Conclusions et perspectives}

L'usinabilité à grande vitesse et à sec des rechargements base-nickel est limitée par l'usure prématurée d'outil. Celle-ci résulte de deux actions :

- action d'adhésion (collage de copeau et écaillement d'arête) ;

- action d'abrasion (labourage et écrouissage).

Ces deux actions sont liées aux caractéristiques microstructurales des couches de rechargement (cinétique d'usure différente entre les couches supérieures et une résistance à l'usure élevée dans la première couche). Les dégradations rapides des outils de coupe dans les couches supérieures poussent à optimiser les conditions de coupe :

1. en favorisant l'évacuation des copeaux et l'utilisation d'un fluide de refroidissement pour diminuer les températures de coupe et l'usure adhésive;

2. en choisissant un engagement axial adéquat pour éviter la couche écrouie;

3. en réduisant la vitesse de coupe et en augmentant la vitesse d'avance pour surmonter la faible résistance au grippage du dépôt ;

4. en recherchant le meilleur compromis entre la géométrie de coupe et le matériau de coupe;

5. en comprenant précisément l'influence de la microstructure (fractions volumiques, tailles et dispersions des phases dendritiques et des particules de métal non fondues) sur l'usinabilité.

Enfin, il est clair que des essais de coupe simulant des opérations d'usinage représentatives de formes gauches (évidement de poches fermées, plongée en pleine matière... ) seront nécessaires par la suite afin d'optimiser la réalisation de formes dans ces couches du rechargement ainsi que le choix des stratégies d'usinage.

Remerciements. Les auteurs remercient le Ministère de la Recherche et le conseil Régional Champagne-Ardenne pour le financement de cette étude dans le cadre du Pôle Mécanique et Matériaux Champagne-Ardenne (Contrat de plan État/Région). Les auteurs remercient également les partenaires de l'étude : les Ateliers des Janves, Castolin France et le CRITT Matériaux, Dépôts et Traitements de Surface (Charleville-Mézières). 


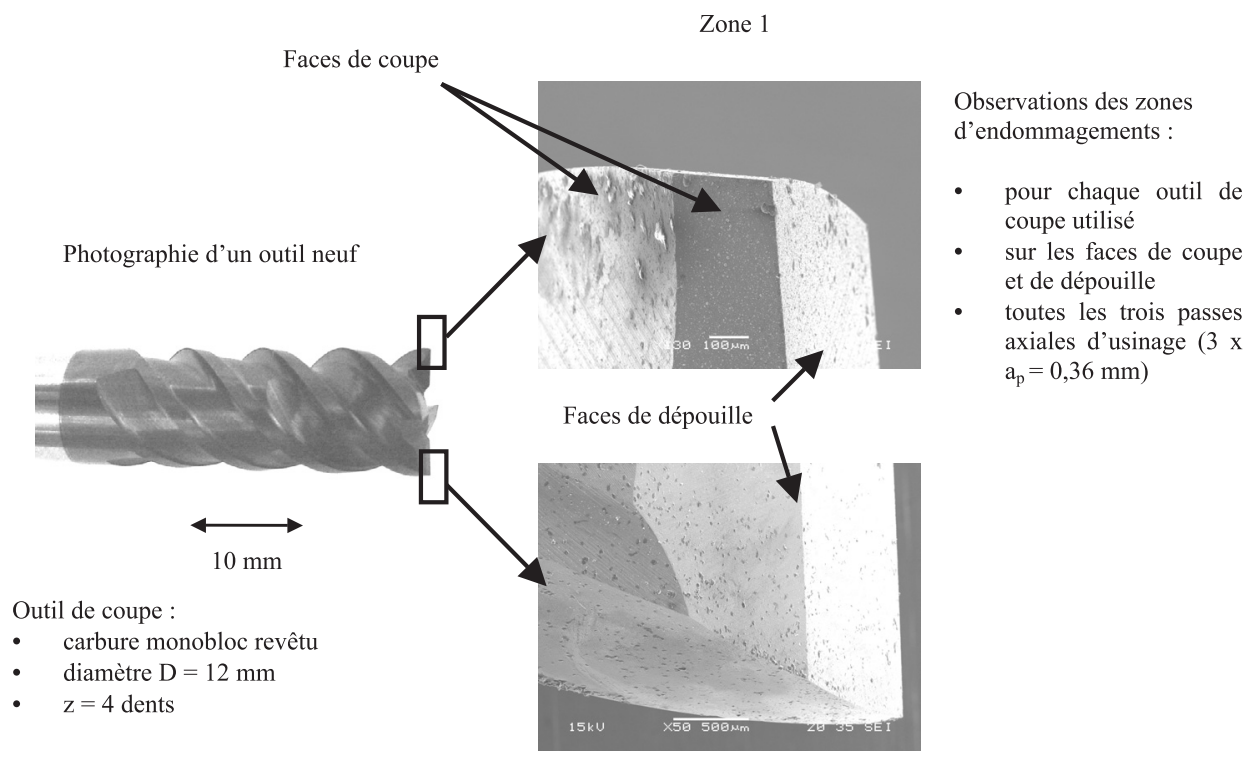

Zone 2

Fig. 13. Zones d'observations des endommagements des outils de coupe au microscope électronique à balayage.

Zone 1

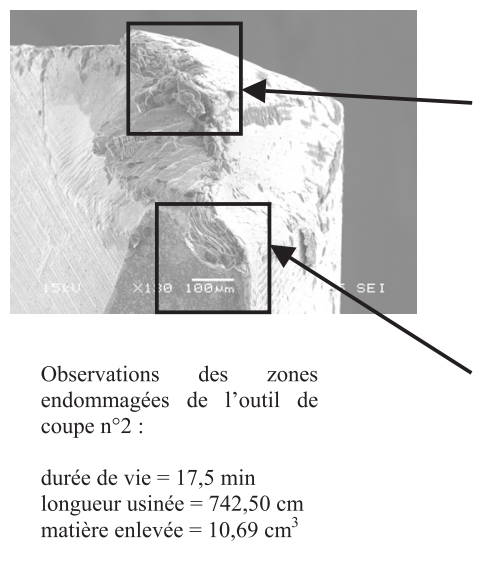

Zone 2

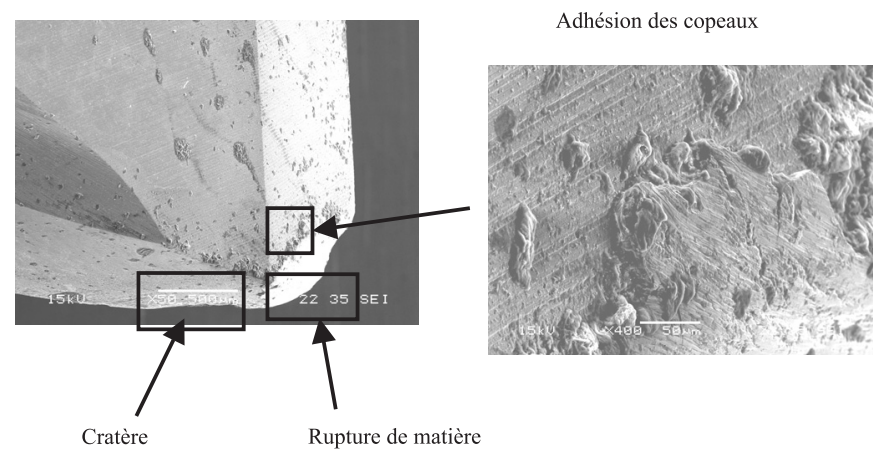

Fig. 14. Endommagements observés sur la face de coupe et sur la face de dépouille $\left(V_{\mathrm{c}}=80 \mathrm{~m} \cdot \mathrm{min}^{-1}, f_{z}=0,05 \mathrm{~mm}\right.$, $\left.a_{\mathrm{e}}=12 \mathrm{~mm}, a_{\mathrm{p}}=0,12 \mathrm{~mm}\right)$.

\section{Références}

[1] IS \& CETIM, Rechargement par soudage des outillages de forge à chaud : Guide pour la rédaction d'un programme de rechargement par soudage à l'arc des outillages de forge à chaud, 1995

[2] E.O. Ezugwu, Z.M. Wang, A.R. Machado, The machinability of nickel-based alloys: a review, J. Materials Processing Technology 86 (1999) 1-16

[3] E.O. Ezugwu, I.R. Pashby, High speed milling of nickelbased superalloys, J. Materials Processing Technology 33 (1992) 429-437

[4] CETIM, Les atouts de l'usinage à grande vitesse : Fraisage et perçage des métaux durs, 1996

[5] T. Kitagawa, A. Kubo, K. Maekawa, Temperature and wear of cutting tools in high-speed machining of Inconel 718 and Ti-6Al-6V-2Sn, wear 202 (1997) 142-148

[6] B.V. Cockerman, The Fracture toughness and Toughening Mechanisms of nickel-base wear materials, J. Metall. Mater. Trans. A 33 (2002) 33-56

[7] ASTM E399-90, Standard Test Method for Plane-Stain Fracture Toughness of Metallic Materials, Annual Book of ASTM Standards, 1990

[8] D. François, L. Joly, La rupture des métaux, Masson et compagnie, 238, 1972

[9] B.V. Cockerman, R.F. Buck, W.L. Wilson, Laboratory galling of several commercial cobalt-free weld hardfacing alloys, Surface and Coatings Technology 94-95 (1997) 495-500

[10] ASTM G98, Standard Test Method for Galling Resistance of Materials, Annual Book of ASTM Standards 03-02 (1992)

[11] X.J. Ren, R.D. James, E.S. Brookes, L. Wang, Machining of high chromium hardfacing materials, J. Materials Processing Technology 115 (2001) 423-429 
[12] AFNOR NF E 66-505, Essai de durée de vie des outils de tournage à partie active unique, NF E 66-505 (1993)

[13] G. Poulachon, Aspects phénoménologiques, mécaniques et métallurgiques en tournage c-BN des aciers durcis, Thèse, École nationale supérieure d'arts et métiers de Cluny, 1999

[14] F. Le Maître, Relations entre énergies de coupe et endommagement des outils de coupe, Wear 62 (1980) 139-160

[15] M.C. Shaw, Metal Cutting Principles, Clarendon Press, Oxford, 1984
[16] R. Komanduri, R.H. Brown, The mechanisms of chip segmentation in machining, ASME J. Engineering for Industry 103 (1981) 33-51

[17] R. Komanduri, T. Schroeder, B.F. Von Turkovich, J. Hazra, D.G. Flom, On the catastrophic shear instability in high speed machining of an AISI4340 steel, ASME J. Engineering for Industry 104 (1982) 121-131

[18] H. Schultz, Fraisage grande vitesse des matériaux métalliques et non métalliques, Société Française d'Éditions techniques SOFETEC, Boulogne, 1997 